\title{
Nanoparticle Type
}

National Cancer Institute

\section{Source}

National Cancer Institute. Nanoparticle Type. NCI Thesaurus. Code C62379.

The classification of discrete nanoparticle entities. 